\title{
Validation of Novel Wobbe Index Sensor for Biogas Cogeneration
}

\author{
Solid State Phenomena 254, pp 43-48 http://hdl.handle.net/10.4028/www.scientific.net/SSP.254.43 \\ Submitted: 2016-01-31 Revised: 2016-04-07 Accepted: 2016-04-07 \\ (C) 2016 Trans Tech Publications, Switzerland Online: 2016-08-30
}

SLATER Conor, FARINE Gaël, MAEDER Thomas, and RYSER Peter, Laboratoire de Production Microtechnique (LPM), Ecole Polytechnique Fédérale de Lausanne (EPFL), Lausanne, Switzerland. conor.slater@epfl.ch, gael.farine@epfl.ch,thomas.maeder@epfl.ch, peter.ryser@epfl.ch

Keywords: Sensor, Wobbe Index, Biogas, Natural Gas, CHP, Cogeneration

\begin{abstract}
Biogas is a fuel made from the anaerobic digestion of organic material to form methane. It can be used to power a stationary engine to generate electricity making it a viable method of decentralised power generation from renewables. However, biogas is a mixture of methane and carbon dioxide, and other trace gases such as hydrogen, hydrogen sulphide and oxygen. As such the quality can vary and setting the air-fuel ratio for efficient combustion can be problematic under these conditions. The Wobbe Index, or Wobbe Number, is a quality of combustible gases that allows the air-fuel requirement to be determined. This work presents a novel type of Wobbe Index sensor based on a miniaturised capillary viscometer that can be used with biogas. The sensor is validated at a biogas cogeneration plant which uses a stationary engine and the results are compared to a methane sensor installed at the plant.
\end{abstract}

\section{Introduction}

Biogas is a fuel made from the anaerobic digestion of organic material to form methane. It can be used to power a stationary engine to generate electricity making it a viable method of decentralised power generation from renewables. It can also be used directly for heating and cooking and if it is upgraded to Biomethane it can be injected into the natural gas grid. However, biogas is a mixture of methane and carbon dioxide, and other trace gases such as hydrogen, hydrogen sulphide and oxygen. As such the quality can vary and setting the air-fuel ratio for efficient combustion can be problematic under these conditions.

The Wobbe Index, or Wobbe Number, is a quality of combustible gases that allows the air-fuel requirement to be determined. It is commonly used as a measure of the interchangeability of gas. Essentially, with the same valve setting for air and fuel requirements, a burner can burn all fuels that have the same Wobbe Index, regardless of the individual composition of the gases.

This work presents a novel type of Wobbe Index sensor based on a miniaturised capillary viscometer that can be used with biogas and natural gas. The sensor is validated at a biogas cogeneration plant which uses a stationary engine and the results are compared to a methane sensor installed at the plant. Before the validation the sensor performance was analysed with a laboratory test setup, where different gasses tested by the sensor. The model for viscosity to Wobbe Index conversion was analyses using existing theoretical models for calculation the Wobbe Index and viscosity of gas mixes. The validation itself was carried out over a month long period where the output of Wobbe Index sensor was compared to an infrared methane sensor.

\section{Theoretical Model}

In this work the methods and constants details in ISO 6976 were used for the calculation of the Wobbe Index. In summary the Wobbe Index can be calculated as in Equation 1. below.

$$
W I=\frac{H H V}{\sqrt{\frac{\rho g a s}{\rho_{\text {air }}}}}
$$


Where, $W I$ is the Wobbe Index of the gas, HHV is its higher heating value, $\rho_{\text {gas }}$ is its density and $\rho_{\text {air }}$ is the density of air.

Equation 2. was derived from [1] and was used to calculate the viscosity.

$$
\eta_{m}=\sum_{i=1}^{v} \frac{x_{i} \eta_{i}}{\sum_{j=1}^{v} x_{j} \phi_{i j}}
$$

With: $\phi_{i j}=\frac{\left[1+\left(\eta_{i} / \eta_{j}\right)^{1 / 2}\left(M_{i} / M_{j}\right)^{1 / 4}\right]^{2}}{\left[8\left(1+M_{i} / M_{j}\right)\right]^{1 / 2}}$

Where, $\eta_{\text {mix }}$ is the dynamic viscosity of the gas mix, $\eta$ is the dynamic viscosity of a component gas $x$ is the molar fraction and $M$ is the molar mass.

\section{Design of Viscometer}

Design of Wobbe Index Sensor is detailed in Figure 1. It is essentially a miniaturized capillary viscometer with the gas being pumped by a ceramic heater and the pressure across the capillary being measured by a MEMS differential pressure sensor. The sensor itself is held in a flow cell and samples the gas as it flows through. To make a measurement the sensor heats the gas contained in the cavity to increase its pressure. The pressurized gas flows out of the cavity through the capillary and pressure inside the cavity decreases in an exponential decay. It is from this time constant that the viscosity of the gas can be calculated. A short time constant means a low viscosity gas and long time constant means a high viscosity gas. After the measurement is taken the heat turns off and the pressure in the cavity decreases and a new sample is drawn in through the capillary. The entire process takes between 90 and 120 seconds. The operation of the sensor is further described in [2].

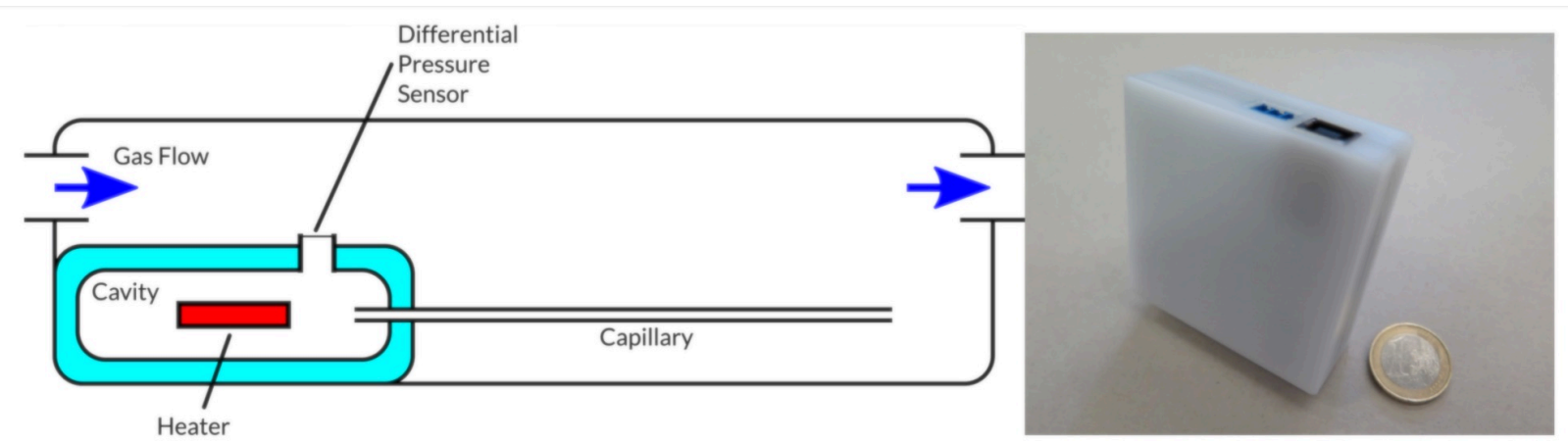

Fig 1. Schematic of Sensor (left), Wobbe Index Sensor (right)

\section{Benchtop Test}

To evaluate the performance of sensor a laboratory test setup was constructed that could mix methane and nitrogen and pass them through the sensor to test for different Wobbe Index values. A schematic of the test setup is detailed in Figure 2. Compressed cylinders of methane and nitrogen are connected through pressure reducing manometers to gas flow controllers (EL-Flow F-201CB, Bronkhorst, Netherlands) calibrated for the respective gases. Flow rate of each of the gases can be adjusted from 0 to $100 \mathrm{ml}$ per minute and they are passed through a static mixer allowing mixes of methane and nitrogen to be sent to the sensor. A variable flow restriction is placed after the sensor to allow the pressure to be regulated and after the gas flows through the sensor is directed to the air extraction. 


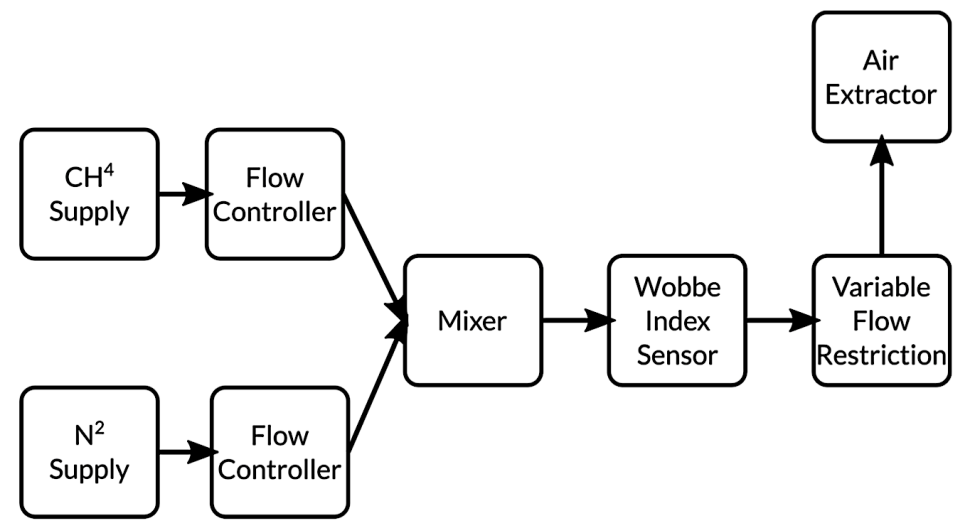

Fig 2. Test Setup

The sensor was initially tested with pure methane (G20) and methane with $14 \%$ nitrogen (G25). The total flow rate was set to $10 \mathrm{ml} / \mathrm{minute}$ for all experiment and the pressure was adjusted to $100 \mathrm{mBar}$ gauge pressure using the variable flow restriction. 10 consecutive measurements were made with each gas and compared to the calculated Wobbe Index. The results of the experiments are shown in Figure 3.

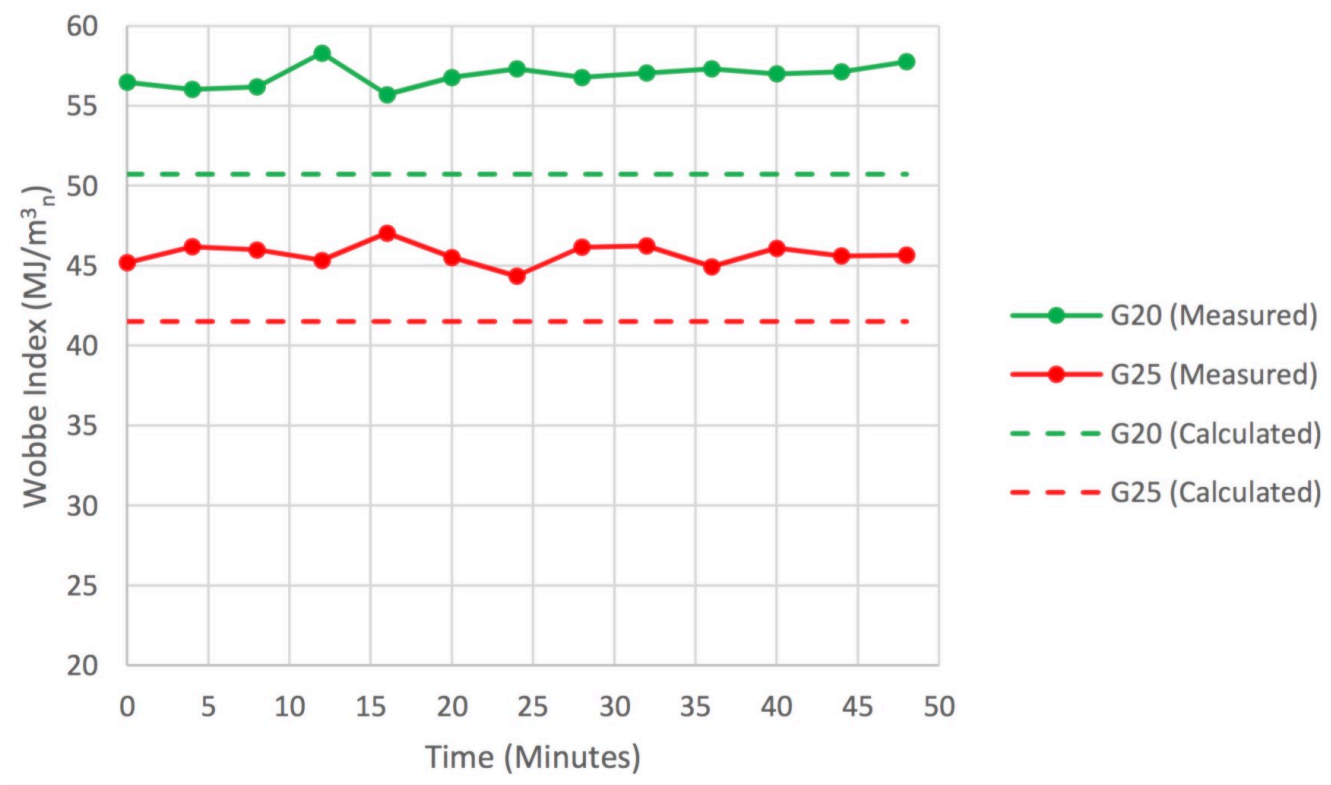

Fig 3. Sensor Test with G20 and G25

For each of the plots there is a clear distinction between the two gas types. The precision was calculated as $\pm 2 \mathrm{MJ} / \mathrm{m}^{3}$ for both data sets and there is and average error of $12 \%$ for G20 and $10 \%$ for G25. The error in the measurement is most likely due to the model used to convert the measured viscosity to the Wobbe Index this was adapted from the model developed in $[3,4]$ (see Equation 3. below).

$$
W I=-102.5496 \cdot \log _{\mathrm{e}}(\eta)-1119.96
$$

To test this hypothesis, the Wobbe Index and viscosity for a number of gas mixes (see Table 1.) were calculated and plotted along with the curve from the model for comparison (see figure 4). It can be seen that there is a consistent error between the model and the calculated values. 


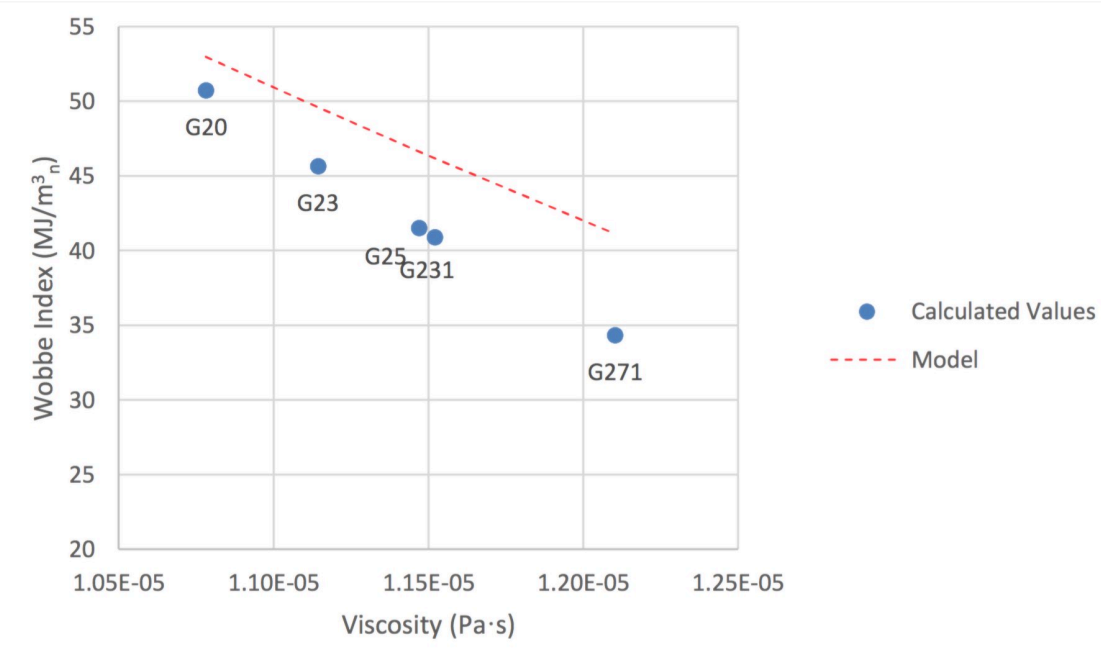

Fig 4. Viscosity to Wobbe Index Conversion

Table 1. Table of Gas Mixtures

\begin{tabular}{|l|l|l|}
\hline Gas & Methane (\%) & Nitrogen (\%) \\
\hline G20 & 100 & \\
\hline G23 & 92.5 & 7.5 \\
\hline G25 & 86 & 14 \\
\hline G231 & 15 & 85 \\
\hline G271 & 74 & 26 \\
\hline
\end{tabular}

\section{Sensor validation}

To determine the function of the Wobbe Index Sensor in a real situation, it was installed a biogas cogeneration plant. The sensor was installed after an infrared methane sensor just before the compressor for the motor (see figure 5.). The methane sensor recorded the daily average methane percentage and the Wobbe Index Sensor was set to record average daily Wobbe Index. The installation remained in place for one month and the data is plotted in figure 6. The Wobbe Index of the Biogas is also calculated from the methane measurements based on the assumption that the remaining percentage is composed entirely of carbon dioxide and is also plotted in figure 6.

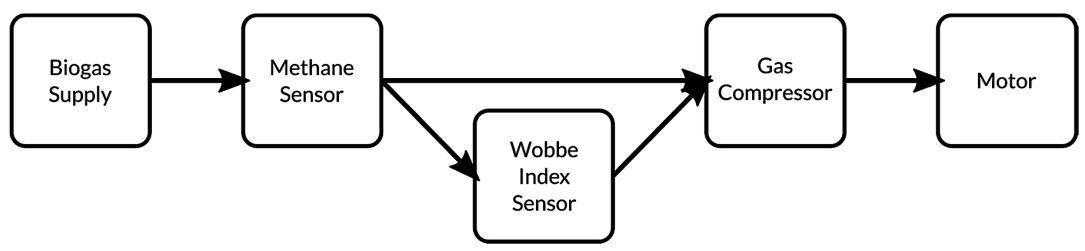

Fig 5. Installation at Biogas Co-Generation Plant

It can be seen in the figure below that the methane percentage, shown in red, stays around $60 \%$ for the duration of the test. The calculated Wobbe Index, shown in green, is determined to be around $23 \mathrm{MJ} / \mathrm{m} 3 \mathrm{n}$ and can be compared to the output of the sensor, shown in blue. There is significantly more variation in the output sensor than in the laboratory test. This may be due to the effect of the gas compressor for the motor, which causes the pressure to vary rapidly in the section of tube. The sensor relying on a flow of gas would be sensitive to this variation as it would cause the flow to change during a measurement. There is also a significant error of approximately $50 \%$ in the Wobbe Index value, this may be caused by the error in the model and also by the presence of the carbon dioxide and other gases such as hydrogen and hydrogen sulphide which the model is not adapted to. 


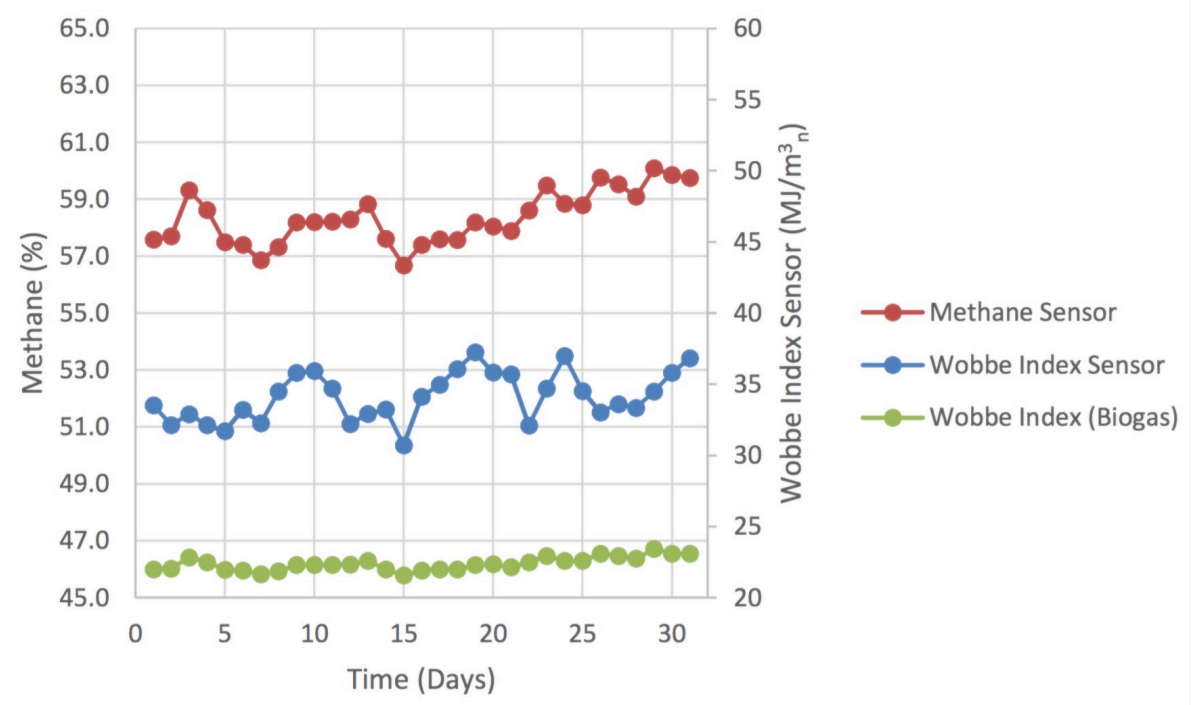

Fig 6. Viscosity to Wobbe Index Conversion

\section{Summary}

In this paper the a Wobbe Index Sensor was validated for a biogas co-generation application. The sensor was first validated in a laboratory setting which is was tested with different gas mixtures. The test showed that with a precision of $\pm 2 \mathrm{MJ} / \mathrm{m} 3 \mathrm{n}$ it could determine the difference between the two gas types. Further analysis showed that there was a significant error in the output and it was demonstrated by theoretical work that the this was due an error in viscosity to Wobbe Index conversion. Subsequently the sensor was installed a biogas co-generation plant to measure the Wobbe Index in series with an infrared methane sensor. The validation showed the sensor had a lower precision due to the gas compressor placed before the motor and there was a significantly increased error, which was probably due to the sensor not being calibrated with high amounts of carbon dioxide. Further work will see an enhanced model for converting the viscosity to the Wobbe Index and methods for reducing the effect of pressure variation on the sensor output. As the biogas cogeneration application can be seen a harsh test the sensor with be further validated for standard natural gas applications where the pressure would be more stable and the amount of carbon dioxide reduced. However, this preliminary test shows that with these improvements the Wobbe Index sensor could have wide variety of applications.

\section{Acknowledgements}

The research was funded by CTI grant number 15614.2 PFEN-NM "Compact Wobbe Index Measurement System Evaluation Kit".

\section{References}

[1] O. Le Corre and K. Loubar, Natural Gas : Physical Properties and Combustion Features, in: P. Potocnik (Eds.), Natural Gas, Sciyo , 2010, pp. 39-70. DOI 10.5772/9823

[2] C. Slater, T.Maeder, P.Ryser, Portable LTCC gas viscometer for determining Wobbe number. Proceedings of Eurosensors 24, Linz, Austria, September 2010. DOI 10.1016/j.proeng.2010.09.109

[3] K. Pickenäcker, D. Trimis, K. Warwzinek, Excess air controlled operation of boilers and furnaces by means of wobbe number measurement. 5th European Conference on Industrial Furnaces and Boilers, Porto, Portugal, April 2000.

[4] K. Warwzinek, D. Trimis, Development of a Wobbe number sensor for controlled combustion of gaseous fuels with varying quality. Proceedings of the 6th International Conference on Technologies and Combustion for a Clean Environment (Clean Air VI), Porto, Portugal, July 2001. 